\title{
ALLELOPATHIC IMPACT OF SORGHUM ON WEED MANAGEMENT, YIELD AND QUALITY OF MAIZE UNDER DIFFERENT SEED RATES
}

Tariq Ahmed Keerio ${ }^{1 *}$, Muhammad Nawaz Kandhro ${ }^{1}$, Ahmed Naqi Shah ${ }^{1}$, Muhammad Ibrahim Keerio ${ }^{2}$, Ghulam Murtaza Jamro ${ }^{3}$

DOI: https://doi.org/10.28941/pjwsr.v27i4.1009

\begin{abstract}
A field study was carried out to estimate the allelopathic potential of sorghum immature and mature plant extracts under various seed rates on weeds density and yield of maize cultivar Dadu Maize-I. The experiment includes different weed control practices i.e., control (No weeding), various levels of sorghum immature and mature plant extracts, herbicides and hand weeding under various seed rates (20, 25, 30 and $35 \mathrm{~kg}$ per ha). The results showed that various weed control practices demonstrated substantial decrease in weeds and caused increase in kernel yield over control. The application of sorghum immature extract @ 15 L per ha in combination with herbicide (Mesotrione Atrazine) @ $1.0 \mathrm{~L}$ per ha decreased effectively weed density $\left(\mathrm{m}^{-2}\right)$ and dry biomass $\left(\mathrm{g} \mathrm{m}^{-}\right.$ ${ }^{2}$ ) and resultantly produced superior growth and yield traits particularly kernel yield ( $t$ per ha) of maize. Sorghum mature plant extract @ $15 \mathrm{~L}$ per ha in integration with herbicide (Mesotrione Atrazine) @ $1.0 \mathrm{~L}$ per ha ranked $2^{\text {nd }}$ in effectiveness of controlling weeds as well as increasing maize kernel yield ( $t$ per ha). As the effect of seed rates is concerned, the least weed density $\left(\mathrm{m}^{-2}\right)$ and greatest kernel yield ( $t$ per ha) were noticed in seed rate $35 \mathrm{~kg}$ per ha, but optimal kernel yield ( $\mathrm{t}$ per ha) was record in seed rate 30 $\mathrm{kg}$ per ha. Hence, the results suggested that combination of plant extracts and herbicide at reduced doses-controlled weeds efficiently and optimum enhancement in kernel yield of maize at a seed rate $30 \mathrm{~kg}$ per ha.
\end{abstract}

Keywords: Allelopathy, Kernel yield, Seed rates, Sorghum extract, Weeds

Citation: Keerio, T.A.; M.N. Kandhro; A.N. Shah; M.I. Keerio; G.M. Jamro. 2021. Allelopathic Impact of Sorghum on Weed Management, Yield and Quality of Maize under Different Seed Rates. Pak. J. Weed Sci. Res., 27(4): 513-526.

\footnotetext{
${ }^{1}$ Department of Agronomy, Sindh Agriculture University, Tandojam, Pakistan ${ }^{2}$ Department of Crop Physiology, Sindh Agriculture University, Tandojam, Pakistan ${ }^{3}$ Department of Soil Science, Sindh Agriculture University, Tandojam, Pakistan *Corresponding author's email: tariqahmedkeerio4@gmail.com
} 
514 Tariq Ahmed Keerio, Muhammad Nawaz Kandhro et al. Allelopathic Impact of ....

\section{Introduction}

Maize (Zea mays L.) is an important cereal crop in Pakistan (Jan et al., 2021). It occupies valuable position in agriculture because it has more potentiality for grain yield and it has short growth period (Ahmad et al., 2018). Maize is the main and important resource of fodder, food and it is used as multipurpose. It is being cultivated in spring as food purpose and in summer for fodder purpose throughout world. Because of this, the demand of maize is increasing rapidly as human population and livestock industry increased significantly. Additionally, oil industries are also in demand of bringing more area under cultivation of maize crop. Due to its short duration growth habit and multipurpose uses, farmers also prefer to grow maize to earn maximum. Maize can be grown in diverse climatic conditions, but it is very complex to low humidity and high temperature and ultimately cause reduction in grain yield (Hashim et al., 2013).

Weeds are one of the significant factors that limit the crop productions as they compete with main crop for moisture, sunlight, space, and nutrients. It is well documented that initial growth and adaption of broader space favours the weeds to grow expensively in the field (Asad et al., 2019). In contrast to environmental effects, weeds are also major sources of yield losses in maize crop (Rajcan and Swanton, 2001). In addition, weeds also interfere with crop plants through allelopathy (Kandhro et al., 2016). However, Karki et al. (2010) reported the $48 \%$ reductions in the grain yield of maize due to the weed invasion in field and further such losses depend on the types of weeds being grown in field. Sharma and Singh (2011) concluded that competition of weeds and crops for water, nutrients and other growing aspects and in lack of an effective weed controlling measures weeds remove large quantity of applied nutrients resulting in more damage yield. Weeds may also lead to higher cost in agriculture production. Small holder farmers spend $50-70 \%$ of their total farm labor economical on suppress to weeds which is mostly by hoe-weeding (Gianessi and Williams, 2011).

Allelopathy is one of the environment friendly and reasonable approaches for weed management (Chopra et al., 2017). Many recent research studies have revealed that noxious weeds can be controlled by using extracts of allelopathic plants (Javaid and Khan, 2020). Allelopathy refers to releasing of chemicals by plant inhibiting the crop growth and development of main crop. It is a mechanism in which chemical produced by weed plants may increase or decrease the associated plant growth (Weston and Duke, 2003). The term allelopathy refers to the interaction between plants and microorganisms as the effects of one plant to other plant through release of chemicals in environments (Weston et al., 2013). These allelochemicals can efficiently be used as to control weeds by planting allelopathic in intercropping, crop rotation, mulching (Farooq et al., 2020). These plants show repressive or seldom stimulatory effects on the germination and growth of the other plants in the immediate vicinity (Cheema et al., 2013). The allelopathic potential of various crops has been identified and used by previous researchers (Cheema et al., 
2009; Mushtaq et al., 2010).

Sorghum is one of the most powerful allelopathic crops which contains several phytotoxic chemicals (Mahmood, 2003). These compounds are the highly water soluble and can be released through the root exudation, from plants via rain and through breakdown of residues (Deka et al., 2011). Sorghum releases sorgoleone, benzoquinone, numerous phenolic, and cyanogenic glycoside allelopathic compounds that actively reduce the growth of nearby plant species (Weston et al., 2013) and affect the germination of seeds (Hozayn et al., 2011) due to highly allelopathic nature. Allelopathy has been effectively utilized to control weeds in various crops including maize fields. Weed density and biomass is reduced significantly by using allopathic crops and weeds (Javaid et al., 2010). The use of the sorghum as allelopathic crop for suppression of weeds is potentially important with regards to the crop productivity, management of ecosystem stability and conservation of genetic diversity (Nouri et al., 2012). However, sorghum material can be used in different ways to control weeds such as surface mulch (Cheema et al., 2004), spray of aqueous extracts (Cheema et al., 2002) crop rotation (Narwal, 2000). Foliar spray of sorghum extract reduced the density and dry weight of purple nutsedge by $44 \%$ and $67 \%$ and increased maize grain yield up to $44 \%$ (Cheema et al., 2004).

High seed rate is an important approach which helps to increase plant population and crop competitiveness against the weeds. More seeding rate generally produces dense plant stand and allows the crop to compete well with weeds (Chauhan and Johnson, 2011). Gill (2008) stated that seed rate facilitates quick canopy closure that helps the suppression of weeds more effectively. However, low seed rate plants usually take extra time to near their canopy which favors the weeds to grow (Arce et al., 2009). Gill (2008) reported more seeding rates helps fast canopy closure which facilitates to decrease weeds more effectively. Numerous studies have been published indicating that increase in crop competitiveness is an important goal while weed control practices. This can be accomplished by reduction of the crop space, adequate seed rate, change of sowing methods, using weed exploitive varieties and valuable applications of the water and nutrients (Bastiaans et al., 2008: Chauhan, 2020). Keeping in view the facts stated above, this study was conducted with the objectives; i.) to examine the weed management under allelopathic effect of sorghum and ii.) to determine the best treatment for obtaining enhanced yield of maize.

\section{MATERIALS AND METHODS}

To examine the weed inhibition and yield improvement in maize through allelopathic action of sorghum immature and mature plant extract in combination with seed rates, the study was laid out at Agriculture Research Center, Tandojam, Pakistan in spring 2019 and 2020. The design of experiment was randomized complete block design having factorial arrangements, three replications and net plot size of $5 \times 4 \mathrm{~m}$ $\left(20 \mathrm{~m}^{2}\right)$. The seed of maize variety Dadu Maize-I was used for sowing purpose. The texture of soil appeared silty clay loam, with limited nitrogen $(0.021 \%)$, low organic matter $(0.42 \%)$ and high in 
516 Tariq Ahmed Keerio, Muhammad Nawaz Kandhro et al. Allelopathic Impact of ....

$\mathrm{pH}$ (8.24). The various seed rates $(20$, $25,30 \& 35 \mathrm{~kg} \mathrm{ha}^{-1}$ ) were tested against various weed control practices, no weeding, Sorghum immature and mature plant extracts at $15 \mathrm{~L}$ per ha alone and combined with reduced doses of herbicide, Herbicide (Mesotrione Atrazine $48 \mathrm{SC}$ ) at $2 \mathrm{~L}$ per ha and Hand weeding twice. Nitrogen and phosphorus were applied at recommended dose of 25-50 kg ha-1 in urea and DAP form.

\section{Weed and crop observations}

Weed density $\left(\mathbf{m}^{-2}\right)$ : Weeds were counted at 60 days after sowing (DAS). A wooden frame of one meter square was utilized to note total number of weeds per meter square.

\section{Weed density $\left(\mathrm{m}^{-2}\right)=\quad$ Total No. of weeds $\left(\mathrm{m}^{-2}\right)$ for given treatment \\ No. of replications}

Weed dry biomass $\left(\mathrm{g} \mathrm{m}^{-2}\right)$ : The weed dry biomass was collected from 3 locations of every unit. Weeds were harvested at floor level and dried up through oven for 48 hours at $70^{\circ} \mathrm{C}$ to note dry weed weight.

Crop stand $\left(\mathbf{m}^{-2}\right)$ : To record this observation crop plants were counted per square meter from three locations of each treatment and averaged.

Leaf area index: The data of leaf area index was noted from selected 5 plants when crop was in peak vegetative growth by the formula:

$$
\text { Leaf area index }=\frac{\text { Leaf area per plant }\left(\mathrm{cm}^{2}\right)}{\text { Ground area per plant }\left(\mathrm{cm}^{2}\right)}
$$

Kernel rows per cob: At the time of maturity, Kernel rows per cob were count from selected plants and average calculate.

Seed index (1000-kernel wt., g): A sample of thousand kernels were taken from each plot and weight on an electric balance.

Biological yield ( $\mathbf{t} \mathbf{h a}^{-1}$ ): Data of crop biological yield was noted as per following formula:

Biological yield $=$ Biological yield plot ${ }^{-1}(\mathrm{~kg}) \div$ Area of plot $\left(\mathrm{m}^{2}\right) \times 10000 \div 1000$

Kernel yield (t per ha) Data regarding kernel yield of maize was noted from harvested plants by using following formula.

Kernel yield $\left(\mathrm{t} \mathrm{ha}^{-1}\right)=$ Kernel yield per plot $(\mathrm{kg}) \div$ Area of plot $\left(\mathrm{m}^{2}\right) \times 10000 \div 1000$

\section{Metrological data}

During experimental season the metrological data of Tandojam 2 years (2019 and 2020) were collected from Metrological Observatory Station, DRIP, 1.
Tandojam. The information of metrological data on monthly based for February, March, April and May about standard temperature $\left({ }^{\circ} \mathrm{C}\right)$, rainfall $(\mathrm{mm})$ and humidity (\%) are mentioned in Fig. 

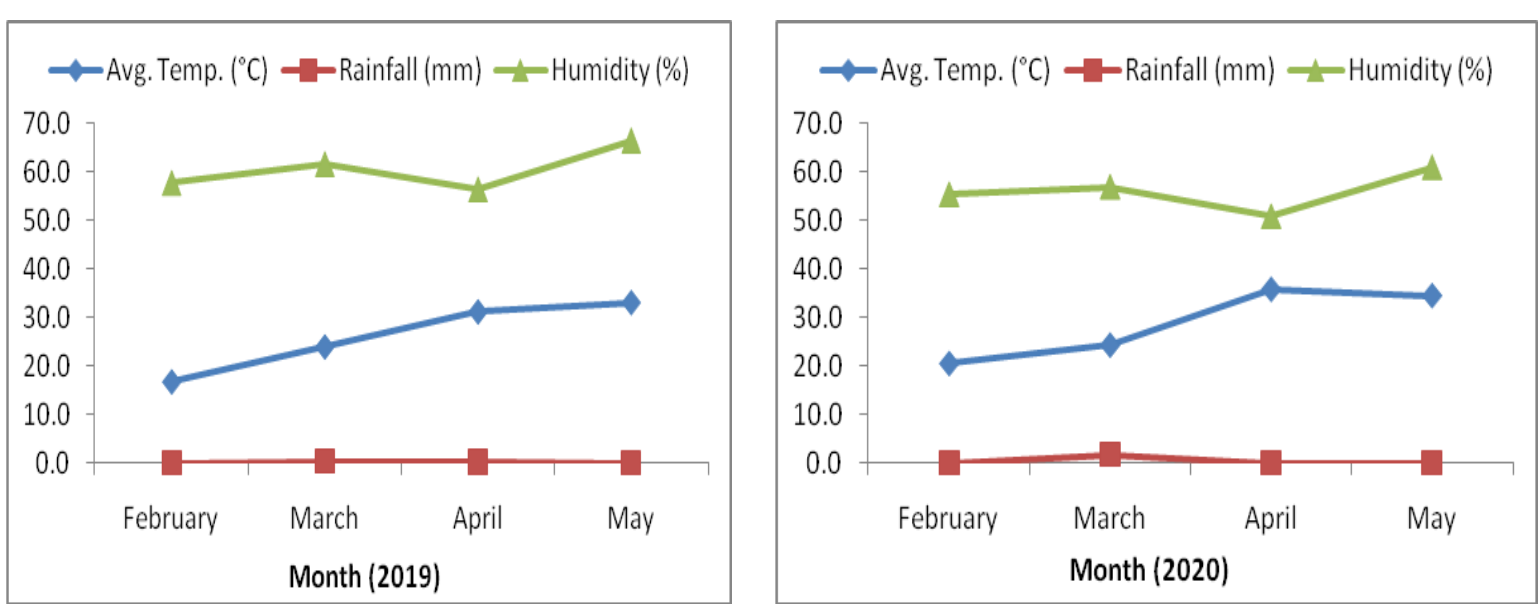

Fig. 1: Meteorological data of Tandojam during crop season (2019 and 2020)

\section{RESULT AND DISCUSSIONS Weed density $\left(\mathbf{m}^{-2}\right)$}

Different weed control practices caused substantial effect on weed density $\left(\mathrm{m}^{-2}\right)$ of maize in contrast to no weeding (Table 1). Integration of sorghum immature extract @ 15 L per ha + herbicide (Mesotrione Atrazine) @ $1.0 \mathrm{~L}$ per ha resulted in lowest $\left(63.5 \mathrm{~m}^{-2}\right)$ weed density followed by integration of sorghum mature plant extract @ 15 L per ha + herbicide (Mesotrione Atrazine) (@) $1.0 \mathrm{~L}$ per ha with weed density of $81.8 \mathrm{~m}^{-2}$. However, highest $\left(171.5 \mathrm{~m}^{-2}\right)$ weed density was noted in No weeding. Seed rates also significantly affected the weed density. The seed rate @ $35 \mathrm{~kg}$ per ha provided least $\left(113.9 \mathrm{~m}^{-2}\right)$ weed density whereas seed rate of $30 \mathrm{~kg}$ per ha trailed in effectiveness resulting in 114.2 weeds $\mathrm{m}^{-2}$ having non-significant statistical differences with each other.
Significant effects for interaction of weed control practices $x$ seed rates were also observed for weed density. These results are link with those of Cheem et al. (2004) who recommended that sorghum releases many allelopathic compounds that influence the development of neighboring flora and they affect germination of seeds (Hozayn et al., 2011). Weed density reduced in the range of 19 to $49 \%$ by using allopathic plants (Mushtaq et al., 2010). Higher seeding rate helps to increase crop competitiveness against weeds (Chauhan and Johnson, 2011). Increased seed rates decreased weed count $\mathrm{m}^{-2}$. Reduce weed density with increasing seed rate might be due to competition from crop plants space, nutrients, moisture and solar energy (Jhala et al., 2008).

Table 1: Weed density $\left(\mathrm{m}^{-2}\right)$ and weed dry weight $\left(\mathrm{g} \mathrm{m}^{-2}\right)$ in maize as affected by sorghum extract under various seed rates

\begin{tabular}{|c|c|c|c|c|c|c|c|c|c|c|}
\hline \multirow[t]{2}{*}{$\begin{array}{l}\text { Weed } \\
(\mathbf{W})\end{array}$} & \multicolumn{4}{|c|}{$\begin{array}{l}\text { Weed density }\left(\mathrm{m}^{-2}\right) \\
\text { Seed rates (kg / ha) }\end{array}$} & \multirow[t]{2}{*}{ Mean } & \multicolumn{4}{|c|}{\begin{tabular}{|c|} 
Weed dry biomass $\left(\mathrm{g} \mathrm{m}^{-2}\right)$ \\
Seed rates $(\mathrm{kg} / \mathrm{ha})$ \\
\end{tabular}} & \multirow[t]{2}{*}{ Mean } \\
\hline & 20 & 25 & 20 & 35 & & 20 & 25 & 20 & 35 & \\
\hline & $\begin{array}{c}174 . \\
8\end{array}$ & 173.9 & 168.8 & 168.3 & $\begin{array}{c}171.5 \\
a\end{array}$ & 532.2 & 513.2 & 494.2 & 475.2 & $\begin{array}{c}503.7 \\
a\end{array}$ \\
\hline IPE (15 L / ha & 142 & 137.2 & 32.5 & 132.3 & 136.0 & 380.2 & 51.2 & 342.8 & 323.2 & 351.7 \\
\hline
\end{tabular}


518 Tariq Ahmed Keerio, Muhammad Nawaz Kandhro et al. Allelopathic Impact of ....

\begin{tabular}{|c|c|c|c|c|c|c|c|c|c|c|}
\hline & & & & & C & & & & & C \\
\hline $\begin{array}{llll}\begin{array}{l}\text { SMPE } \\
\text { ha) }\end{array} & \left(\begin{array}{llll}15 & \text { L } & / \\
\end{array}\right. \\
\end{array}$ & $\begin{array}{c}160 . \\
1\end{array}$ & 155.4 & 149.8 & 149.5 & $\begin{array}{c}153.7 \\
b\end{array}$ & 456.2 & 437.2 & 418.2 & 399.2 & $\begin{array}{c}427.7 \\
b\end{array}$ \\
\hline $\begin{array}{l}\text { Herbicide (2 L / } \\
\text { ha) }\end{array}$ & $\begin{array}{c}105 . \\
5\end{array}$ & 101.6 & 96.0 & 95.3 & $99.9 \mathrm{e}$ & 228.2 & 209.1 & 190.4 & 171.1 & $\begin{array}{c}199.6 \\
\mathrm{e}\end{array}$ \\
\hline $\begin{array}{l}\text { HW } \\
\text { DAS })\end{array}\left(\begin{array}{lll}30 & \& & 55 \\
\end{array}\right.$ & $\begin{array}{c}123 . \\
9\end{array}$ & 119.1 & 114.4 & 114.2 & $\begin{array}{c}117.9 \\
d\end{array}$ & 304.2 & 285.2 & 266.6 & 247.2 & $\begin{array}{c}275.7 \\
d\end{array}$ \\
\hline $\begin{array}{l}\text { SIPE (15 L / ha) } \\
+ \text { Herbicide (1 L } \\
\text { /ha) }\end{array}$ & 69.4 & 65.1 & 59.9 & 59.5 & $63.5 \mathrm{~g}$ & 76.1 & 58.3 & 38.3 & 25.8 & $49.6 \mathrm{~g}$ \\
\hline 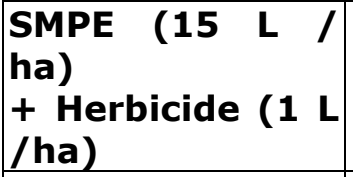 & 87.6 & 83.6 & 78.2 & 77.9 & $81.8 \mathrm{f}$ & 152.1 & 133.1 & 114.1 & 95.1 & $123.6 \mathrm{f}$ \\
\hline Mean & $\begin{array}{c}123 \\
3 a\end{array}$ & $\begin{array}{c}119.4 \\
b\end{array}$ & \begin{tabular}{|c|}
114.2 \\
$c$
\end{tabular} & $\begin{array}{c}113.9 \\
\mathrm{C}\end{array}$ & - & \begin{tabular}{|c|}
304.2 \\
$a$
\end{tabular} & $\begin{array}{c}285.3 \\
b\end{array}$ & $\begin{array}{c}266.2 \\
c\end{array}$ & $\begin{array}{c}248.1 \\
d\end{array}$ & - \\
\hline Variables & \multicolumn{2}{|c|}{ P-Value } & SE & \multicolumn{2}{|c|}{ LSD (5\%) } & \multicolumn{2}{|c|}{ P-Value } & \multicolumn{2}{|l|}{ SE } & LSD (5\%) \\
\hline Weed Practices & \multirow{2}{*}{\multicolumn{2}{|c|}{$0.0000 * *$}} & 0.260 & \multicolumn{2}{|c|}{0.5226} & \multicolumn{2}{|c|}{$0.0000 * *$} & 0.4521 & \multicolumn{2}{|c|}{0.9064} \\
\hline Seed rates & \multirow{2}{*}{\multicolumn{2}{|c|}{$\frac{0.0000 * *}{0.0001 * *}$}} & 0.197 & \multirow{2}{*}{\multicolumn{2}{|c|}{$\begin{array}{l}0.3950 \\
1.0451\end{array}$}} & \multirow{2}{*}{\multicolumn{2}{|c|}{$\begin{array}{l}0.0000 * * \\
0.0002 * *\end{array}$}} & 0.3417 & \multicolumn{2}{|c|}{0.6851} \\
\hline $\mathbf{W} \times \mathbf{S}$ & & & 0.521 & & & & & 0.9041 & & .8127 \\
\hline
\end{tabular}

NW $=$ No weeding, SIPE $=$ Sorghum immature plant extract, SMPE=Sorghum mature plant extract, $\mathrm{HW}=$ Hand weeding, $\mathrm{NS}=$ Non-significant at $\mathrm{P}_{0.05}$ : $*$ Significant at $\mathrm{P}_{0.05} ; * *=$ Significant at $\mathrm{P}_{0.01}$.

\section{Weed dry biomass $\left(\mathrm{g} \mathrm{m}^{-2}\right)$}

All treatments significantly decrease weed biomass compared with No weeding (Table 1). The data revealed that the mixture of sorghum immature extract @ 15 L per ha + herbicide (Mesotrione Atrazine) @ 1.0 L per ha provided reduced $\left(49.6 \mathrm{~g} \mathrm{~m}^{-2}\right)$ weed dry weight followed by incorporation of mature sorghum extract @ 15 L per ha + herbicide (Mesotrione Atrazine) @ $1.0 \mathrm{~L}$ per ha resulting in $123.6 \mathrm{~g} \mathrm{~m}^{-2}$ weed dry biomass against weed dry of $503.7 \mathrm{~g} \mathrm{~m}^{-2}$ documented in no weeding. Seed rates also significantly affected the weed dry biomass. The seed rates $35 \mathrm{~kg} \mathrm{ha}^{-1}$ distinguished the minimum (248.1 $\mathrm{g} \mathrm{m}^{-2}$ ) weed dry biomass followed by seed rate $30 \mathrm{~kg} \mathrm{ha}^{-1}$ with $266.2 \mathrm{~g} \mathrm{~m}^{-2}$ while maximum ( $304 \mathrm{~g} \mathrm{~m}^{-2}$ ) weed dry biomass was noted in seed rate $20 \mathrm{~kg} \mathrm{ha}{ }^{-1}$. Substantial interactive effect of weed control practices $\times$ seed rates was also documented for weed dry biomass. These results concur to the previous studies (Cheema et al., 2004; Khaliq et al., 2012) who described that combine application of extracts with decreased dose of herbicide effectively suppressed weeds and better growth and yield of maize. Mahmood (2009) suggested that allelochemicals found in sorghum residues inhibited weeds more effectively, and resultantly maize growth, yield contributing characters and yield was enhanced due to less opposition of weeds with main crop for inputs. Improved crop population had strong and constant negative effects on weed dry weight. Crop-weed competitiveness is effect by the source availability like mineral nutrient status of soil and the evidence of major nutrients by weeds might be an important tool in application of fertilizers (Yadav et al., 2001) 


\section{Crop stand $\left(\mathbf{m}^{-2}\right)$}

Various weed control practices reflected a positive influence on crop stand of maize (Table 2 ). The maximum $\left(7.7 \mathrm{~m}^{-2}\right)$ crop stand was noted under the mixture of sorghum immature extract @ $15 \mathrm{~L} \mathrm{ha}^{-}$ $1+$ herbicide (Mesotrione Atrazine) @ $1.0 \mathrm{~L}$ per ha trailed by integration of sorghum mature extract @ $15 \mathrm{~L} \mathrm{ha}^{-1}+$ herbicide (Mesotrione Atrazine) @ $1.0 \mathrm{~L}$ ha-1 with $7.4 \mathrm{~m}^{-2}$ crop stand. However, minimum $\left(5.4 \mathrm{~m}^{-2}\right)$ crop stand was found under No weeding. Among seed rates, $35 \mathrm{~kg}$ per ha yielded maximum $\left(7.5 \mathrm{~m}^{-2}\right)$ crop stand. Seed rate $30 \mathrm{~kg}$ per ha proved $2^{\text {nd }}$ in performance resulting in statistically equal crop stand $\left(7.3 \mathrm{~m}^{-2}\right)$. Remarkable effect of interaction of weed control practices $x$ seed rates was noted for crop stand $\left(\mathrm{m}^{-}\right.$ ${ }^{2}$ ). The extracts of different allelopathic crops determinant considerable in growth and development of summer weeds (Kandhro et al., 2015). Our findings are in concurrence with Stougaard and Xue (2004) who concluded that seeding rate was more important for increasing crop density.

Table 2: Crop stand $\left(\mathrm{m}^{-2}\right)$ and leaf area index of maize as affected by sorghum extract under different seed rates

\begin{tabular}{|c|c|c|c|c|c|c|c|c|c|c|}
\hline \multirow{2}{*}{$\begin{array}{l}\text { Weed Practices } \\
\text { (W) }\end{array}$} & \multicolumn{4}{|c|}{$\begin{array}{l}\text { Crop stand }\left(\mathrm{m}^{-2}\right) \\
\text { Seed rates }\left(\mathrm{kg} \mathrm{ha}^{-1}\right)\end{array}$} & \multirow[t]{2}{*}{ Mean } & \multicolumn{4}{|c|}{$\begin{array}{c}\text { Leaf area index } \\
\text { Seed rates }\left(\mathrm{kg} \mathrm{ha}^{-1}\right)\end{array}$} & \multirow[t]{2}{*}{ Mean } \\
\hline & 20 & 25 & 30 & 35 & & 20 & 25 & 30 & 35 & \\
\hline No weeding & 4.5 & 4.8 & 6.0 & 6.2 & $5.4 \mathrm{e}$ & 0.5 & 0.5 & 0.5 & 0.5 & $0.6 \mathrm{~g}$ \\
\hline SIPE (15 L ha-1) & 4.9 & 5.1 & 6.7 & 6.9 & $5.9 \mathrm{~d}$ & 1 & 1.1 & 1.2 & 1.2 & $1.1 \mathrm{e}$ \\
\hline SMPE (15 L ha-1) & 4.8 & 4.9 & 6.1 & 6.3 & $5.5 \mathrm{de}$ & 0.8 & 0.8 & 1 & 1.1 & $0.9 \mathrm{f}$ \\
\hline $\begin{array}{l}\text { Herbicide (2 L ha- } \\
1 \text { ) }\end{array}$ & 5.4 & 6.8 & 8.0 & 8.2 & $7.1 \mathrm{~b}$ & 1.4 & 1.5 & 1.6 & 1.7 & $1.6 \mathrm{c}$ \\
\hline $\begin{array}{l}\text { HW } \\
\text { DAS })\end{array}\left(\begin{array}{lll}30 & \& & 55 \\
\end{array}\right.$ & 5.2 & 6.6 & 7.5 & 7.7 & $6.8 \mathrm{c}$ & 1.3 & 1.3 & 1.4 & 1.5 & $1.4 \mathrm{~d}$ \\
\hline $\begin{array}{l}\text { SIPE }\left(15 \mathrm{Lha}^{-1}\right) \\
+ \text { Herbicide }(1 \mathrm{~L} \\
\left.\mathrm{ha}^{-1}\right)\end{array}$ & 6.8 & 7.2 & 8.3 & 8.5 & $7.7 \mathrm{a}$ & 2 & 2 & 2.2 & 2.2 & $2.1 \mathrm{a}$ \\
\hline $\begin{array}{l}\text { SMPE (15 L / ha) } \\
+ \text { Herbicide ( } 1 \text { L } \\
\left.h^{-1}\right)\end{array}$ & 6.3 & 6.9 & 8.2 & 8.4 & $7.4 \mathrm{a}$ & 1.8 & 1.8 & 1.9 & 2 & $1.9 \mathrm{~b}$ \\
\hline Mean & $5.4 \mathrm{~d}$ & $6.0 \mathrm{c}$ & $7.3 \mathrm{~b}$ & $7.5 \mathrm{a}$ & - & $1.3 \mathrm{c}$ & $1.3 \mathrm{c}$ & $1.4 \mathrm{~b}$ & $1.5 \mathrm{a}$ & - \\
\hline Variables & $\mathrm{P}-\mathrm{Va}$ & alue & SE & & $(5 \%)$ & $P-V a$ & Iue & SE & & SD (5\%) \\
\hline Weed Practices & 0.000 & $00 * *$ & 0.170 & & 317 & 0.000 & $0 * *$ & 0.03 & & 0.0611 \\
\hline Seed rates & 0.000 & $00 * *$ & $0.128 \varepsilon$ & & 2583 & 0.000 & $0 * *$ & 0.023 & & 0.0442 \\
\hline $\mathbf{W} \times \mathbf{S}$ & 0.16 & 665 & $0.340 \varepsilon$ & & 6834 & 0.000 & $0 * *$ & 0.060 & & 0.1221 \\
\hline
\end{tabular}

$\mathrm{NW}=$ No weeding, SIPE $=$ Sorghum immature plant extract, SMPE=Sorghum mature plant extract, $\mathrm{HW}=\mathrm{Hand}$ weeding, $\mathrm{NS}=$ Non-significant at $\mathrm{P}_{0.05} ; *=$ Significant at $\mathrm{P}_{0.05} ; * *=$ Significant at $\mathrm{P}_{0.01}$.

\section{Leaf area index}

This data (Table 2 ) revealed that various weed control practices significantly improved leaf area index of maize as compared to no weeding. The data of leaf area index was noted high (2.1) in plot under combined application of sorghum immature plant extract @ $15 \mathrm{~L}$ $\mathrm{ha}^{-1}+$ herbicide (Mesotrione Atrazine 1.0 $L \mathrm{ha}^{-1}$ ) followed by integration of sorghum mature plant extract @ 15 L ha $^{-1}+$ herbicide (Mesotrione Atrazine 1.0 
L ha-1 ${ }^{-1}$ and (1.9) \& low (0.6) was noticed in no weeding. The data of the effects of seeding rates applications on leaf area performance showed that increasing sowing density from 20 to $35 \mathrm{~kg} \mathrm{ha}^{-1}$ showed increasing trends of leaf area index from 1.3 to 1.5 , respectively. Significant differences among leaf area indexes were noted by weed control practices $x$ seed rates interaction. This may be found by reduce light interface and net assimilation rate depending on precise plant morphology. Significant interaction of weed control practices $x$ seed rates were also observed for leaf area index. Valadabadi and Farahani, (2010) stated that maximum physiological development indices are achieved under more plant population because photosynthesis better by growth of leaf area. Growing plant compression enhanced leaf area index on account area engaged by green canopy of plants per unit area, more capture of solar radiation within the canopy and accumulation of dry biomass (Sharifi and Namvar, 2016).

\section{Kernel rows per cob}

The data (Table 3 ) regarding kernel rows $\mathrm{cob}^{-1}$ showed significant effect of weed control practices. Integration of sorghum immature plant extract @ $15 \mathrm{~L} \mathrm{ha}^{-1}+$ herbicide (Mesotrione Atrazine $1.0 \mathrm{~L} \mathrm{ha}^{-1}$ ) gave significantly highest kernel rows $\mathrm{cob}^{-1} 15.2$ followed by integration of sorghum mature plant extract @ 15 L ha ${ }^{-1}+$ herbicide (Mesotrione Atrazine) @ $1.0 \mathrm{~L} \mathrm{ha}^{-1}$ which produced (14.7) whereas lowest $(10.0)$ kernel rows $\mathrm{cob}^{-1}$ were obtained in no weeding. It is also noticeable that seed rates influenced significantly on kernel rows per cob. The seed rate $20 \mathrm{~kg} \mathrm{ha}^{-1}$ produced more (13.8) kernel rows cob $^{-1}$ followed by seed rate $25 \& 30 \mathrm{~kg} \mathrm{ha}^{-1}$ with 13.2 and 12.3 kernel rows $\mathrm{cob}^{-1}$. Nonetheless, least 11.9 kernel rows cob $^{-1}$ were noted in seed rate $35 \mathrm{~kg} \mathrm{ha}^{-1}$. The interface of weed control practices $\times$ seed rates were found noteworthy for kernel rows per cob. Amiri et al. (2014) described that plant population had a substantial effect on kernels per cob. The kernel rows per cob reduced the weed interference period enlarged. Increase in kernel per cob at lower plant density might be due to lesser opposition that energy and nutrient to allow plants more photosynthesis into sink (Sharifi et al., 2009).

Table 3. Kernel rows $\mathrm{cob}^{-1}$ and seed index ( $\mathrm{g}$ ) of maize as affected by sorghum extract under different seed rates

\begin{tabular}{|c|c|c|c|c|c|c|c|c|c|c|}
\hline \multirow[t]{3}{*}{ Weed Practices (W) } & \multicolumn{4}{|c|}{ Kernel rows cob ${ }^{-1}$} & \multirow[t]{3}{*}{ Mean } & \multirow{2}{*}{\multicolumn{4}{|c|}{\begin{tabular}{|c|}
$\begin{array}{c}\text { Seed index }(1000-k e r n e l \\
\text { wt., } \mathrm{g})\end{array}$ \\
Seed rates $\left(\mathrm{kg} \mathrm{ha}^{-1}\right)$ \\
\end{tabular}}} & \multirow[t]{3}{*}{ Mean } \\
\hline & \multicolumn{4}{|c|}{ Seed rates $\left(\mathrm{kg} \mathrm{ha}^{-1}\right)$} & & & & & & \\
\hline & 20 & 25 & 30 & 35 & & 20 & 25 & 30 & 35 & \\
\hline No weeding & 10.3 & 10.2 & 10.0 & 9.6 & $10.0 \mathrm{~g}$ & 211.3 & 208.3 & 207.5 & 203.0 & $\begin{array}{c}207.5 \\
\mathrm{~g}\end{array}$ \\
\hline SIPE (15 L ha-1) & 13.2 & 12.5 & 10.4 & 10 & $11.5 \mathrm{e}$ & 259.5 & 244.7 & 240.3 & 237.1 & $\begin{array}{c}245.4 \\
\mathrm{e}\end{array}$ \\
\hline SMPE (15 L ha-1) & 11.8 & 10.6 & 10.3 & 9.9 & $10.7 \mathrm{f}$ & 234.5 & 220.5 & 218.2 & 220.2 & $\begin{array}{c}223.3 \\
f\end{array}$ \\
\hline Herbicide (2 L ha-1) & 15.1 & 14.8 & 13.5 & 13.1 & $14.1 \mathrm{c}$ & 312.2 & 298.3 & 284.6 & 286.4 & $\begin{array}{c}295.4 \\
\text { C }\end{array}$ \\
\hline HW (30 \& 55 DAS) & 14.4 & 13.6 & 12.7 & 12.3 & $13.3 \mathrm{~d}$ & 289.1 & 280.4 & 271.4 & 261.8 & 275.7 \\
\hline
\end{tabular}




\begin{tabular}{|c|c|c|c|c|c|c|c|c|c|c|c|}
\hline & & & & & & & & & & & $d$ \\
\hline $\begin{array}{l}\text { SIPE }\left(15 \mathrm{~L} \mathrm{ha}^{-1}\right) \\
+\quad \text { Herbicide }(1 \quad \mathrm{~L} \\
\text { ha-1) }^{-1}\end{array}$ & 16.4 & 15.6 & 14.6 & 14.1 & $15.2 \mathrm{a}$ & 355.8 & 342.0 & 319.6 & \multicolumn{2}{|c|}{316.5} & $\begin{array}{c}333.5 \\
a\end{array}$ \\
\hline $\begin{array}{l}\left.\text { SMPE (15 L ha' }{ }^{-1}\right) \\
+ \text { Herbicide }(1 \text { L ha- } \\
\text { 1) }\end{array}$ & 15.3 & 14.8 & 14.5 & 14.2 & $14.7 \mathrm{~b}$ & 328.7 & 318.2 & 311.4 & \multicolumn{2}{|c|}{307.1} & $\begin{array}{c}316.3 \\
b\end{array}$ \\
\hline Mean & \begin{tabular}{|c|}
$\begin{array}{c}13.8 \\
a\end{array}$ \\
\end{tabular} & $\begin{array}{c}13.2 \\
b\end{array}$ & $12.3 \mathrm{c}$ & $\begin{array}{c}11.9 \\
c\end{array}$ & - & \begin{tabular}{|c|}
284.4 \\
$a$
\end{tabular} & $\begin{array}{c}273.2 \\
b\end{array}$ & $\begin{array}{c}264.7 \\
c\end{array}$ & \multicolumn{2}{|c|}{$261.7 \mathrm{c}$} & - \\
\hline Variables & P-value & & SE & \multicolumn{2}{|c|}{ LSD $(5 \%)$} & \multicolumn{2}{|c|}{ P-value } & \multicolumn{2}{|c|}{ SE } & \multicolumn{2}{|c|}{ LSD (5\%) } \\
\hline Weed Practices & $\begin{array}{c}0.0000 \\
* *\end{array}$ & \multicolumn{2}{|c|}{0.1877} & \multicolumn{2}{|c|}{0.3763} & \multicolumn{2}{|c|}{$0.0000 * *$} & \multicolumn{2}{|c|}{2.7266} & \multicolumn{2}{|c|}{5.4664} \\
\hline Seed rates & 0.0000 & \multicolumn{2}{|c|}{0.1419} & \multicolumn{2}{|c|}{0.3695} & \multicolumn{2}{|c|}{$0.0000 * *$} & \multicolumn{2}{|c|}{2.0611} & \multicolumn{2}{|c|}{4.1322} \\
\hline $\mathbf{W} \times \mathbf{S}$ & $\begin{array}{c}0.0576 \\
* *\end{array}$ & \multicolumn{2}{|c|}{0.3754} & \multicolumn{2}{|c|}{0.7527} & \multicolumn{2}{|c|}{$0.0137 * *$} & \multicolumn{2}{|c|}{5.4531} & \multicolumn{2}{|c|}{10.933} \\
\hline
\end{tabular}

$\mathrm{NW}=$ No weeding, SIPE $=$ Sorghum immature plant extract, SMPE=Sorghum mature plant extract, $\mathrm{HW}=$ Hand weeding, $\mathrm{NS}=$ Non-significant at $\mathrm{P}_{0.05} ; *=$ Significant at $\mathrm{P}_{0.05} ; * *=$ Significant at $\mathrm{P}_{0.01}$.

\section{Seed index (1000-kernel wt., g)}

The cooperation of immature sorghum plant extract @ $15 \mathrm{~L} \mathrm{ha}^{-1}+$ herbicide (Mesotrione Atrazine) @ $1.0 \mathrm{~L} \mathrm{ha}^{-1}$ revealed significantly greatest seed index of $(333.5 \mathrm{~g})$. The integration of sorghum mature plant extract @ 15 L ha $^{-1}+$ herbicide (Mesotrione Atrazine) @ $1.0 \mathrm{~L} \mathrm{ha}^{-1}$ followed and created seed index of $316.3 \mathrm{~g}$ and lowest (207.5) $\mathrm{g}$ seed index was achieved in no weeding. Seed index was significant regarding seed rates effects. Seed rate $20 \mathrm{~kg}$ per ha resulted in more seed index $(284.4 \mathrm{~g})$ followed by other seed rates which produced seed index of 273.2 and 264.7 g. However, less $261.7 \mathrm{~g}$ seed index was observed in seed rate $35 \mathrm{~kg} \mathrm{ha}{ }^{-1}$. The connection of weed control practices $x$ seed rates were found substantial for seed index (Table 3). Thousand kernels weight decreased as the weed interference period increased (Gozubenli et al., 2004).

\section{Biological yield (t per ha)}

The weed control practices gave prominent $(P<0.05)$ results for biological yield of maize (Table 4). The more (17.6 $\mathrm{t} \mathrm{ha}^{-1}$ ) biological yield was found under the association of sorghum immature plant extract @ $15 \mathrm{~L} \mathrm{ha}^{-1}+$ herbicide (Mesotrione Atrazine) @ $1.0 \mathrm{~L} \mathrm{ha}^{-1}$ followed by integration of sorghum mature plant extract @ $15 \mathrm{~L} \mathrm{ha}^{-1}+$ herbicide (Mesotrione Atrazine) @ $1.0 \mathrm{~L}$ ha $^{-1}$ resulting in $17.2 \mathrm{t}$ per ha and lowest $14.5 \mathrm{t} \mathrm{ha}^{-1}$ biological production was achieved in no weeding. Seed rate $35 \mathrm{~kg}$ per ha provided maximum (16.5 $\mathrm{t} \mathrm{ha}^{-1}$ ) biological yield as compared to other seed rates such as 20,25 and $30 \mathrm{~kg} \mathrm{ha}^{-1}$ $\left(15.8,16.2\right.$ and $16.5 \mathrm{t} \mathrm{ha}^{-1}$ ). The interactive effects of weed control practices $\times$ seed rates were found significant for biological yield $t$ per ha. These findings are equal to Khaliq et al. (2012) who reported that combined application of extracts with reduced dose of herbicide effectively suppressed weeds and improved growth and yield of maize. 
522 Tariq Ahmed Keerio, Muhammad Nawaz Kandhro et al. Allelopathic Impact of ....

Table 4: Biological yield ( $\mathrm{t} \mathrm{ha} \mathrm{a}^{-1}$ ) and kernel yield $\left(\mathrm{t} \mathrm{ha} \mathrm{h}^{-1}\right.$ ) of maize as affected by sorghum extract under different seed rates

\begin{tabular}{|c|c|c|c|c|c|c|c|c|c|c|}
\hline \multirow{3}{*}{$\begin{array}{l}\text { Weed } \\
\text { (W) }\end{array}$} & \multirow{2}{*}{\multicolumn{4}{|c|}{\begin{tabular}{|c|}
$\left.\begin{array}{c}\text { Biological yield (t ha- } \\
1\end{array}\right)$ \\
Seed rates $\left(\mathrm{kg} \mathrm{ha}^{-1}\right)$ \\
\end{tabular}}} & \multirow[t]{3}{*}{ Mean } & \multicolumn{4}{|c|}{ Kernel yield (t ha-1) } & \multirow[t]{3}{*}{ Mean } \\
\hline & & & & & & \multicolumn{4}{|c|}{ Seed rates $\left(\mathrm{kg} \mathrm{ha}^{-1}\right)$} & \\
\hline & 20 & 25 & 30 & 35 & & 20 & 25 & 30 & 35 & \\
\hline No weeding & 13.6 & 14.7 & 14.7 & 14.9 & $14.5 \mathrm{~g}$ & 4.1 & 4.1 & 4.2 & 4.3 & $4.2 \mathrm{~g}$ \\
\hline SIPE (15 L ha-1) & 15.8 & 15.3 & 15.8 & 16.3 & $15.8 \mathrm{e}$ & 4.7 & 5.2 & 5.3 & 5.1 & $5.1 \mathrm{e}$ \\
\hline SMPE (15 L ha-1) & 13.8 & 15.2 & 15.4 & 15.7 & $14.9 \mathrm{f}$ & 4.6 & 4.7 & 4.8 & 5.1 & $4.8 \mathrm{f}$ \\
\hline $\begin{array}{l}\text { Herbicide (2 L ha- } \\
\text { 1) }\end{array}$ & 16.5 & 16.8 & 16.9 & 17.0 & $16.8 \mathrm{c}$ & 5.7 & 6.1 & 6.4 & 6.5 & $6.2 \mathrm{c}$ \\
\hline 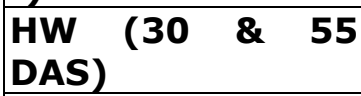 & 16.3 & 16.7 & 16.9 & 16.6 & $16.6 \mathrm{~d}$ & 5.4 & 5.7 & 5.8 & 6.0 & $5.7 \mathrm{~d}$ \\
\hline $\begin{array}{l}\text { SIPE }\left(15 \mathrm{~L} \mathrm{ha}^{-1}\right) \\
+ \text { Herbicide }(1 \mathrm{~L} \\
\left.\mathrm{ha}^{-1}\right)\end{array}$ & 17.4 & 17.5 & 17.6 & 17.8 & $17.6 \mathrm{a}$ & 6.4 & 6.6 & 6.9 & 7.1 & $6.8 \mathrm{a}$ \\
\hline $\begin{array}{l}\text { SMPE (15 L / ha) } \\
+ \text { Herbicide (1 L } \\
\left.\text { ha }^{-1}\right)\end{array}$ & 16.9 & 17.6 & 17.2 & 17.2 & $17.2 \mathrm{~b}$ & 6.2 & 6.3 & 6.4 & 6.5 & $6.4 \mathrm{~b}$ \\
\hline Mean & $\begin{array}{c}15.8 \\
\mathrm{C}\end{array}$ & $\begin{array}{c}16.2 \\
C\end{array}$ & $\begin{array}{c}16.5 \\
a\end{array}$ & $16.5 \mathrm{a}$ & - & $5.3 \mathrm{~d}$ & $5.5 \mathrm{c}$ & $5.7 \mathrm{~b}$ & $5.8 \mathrm{a}$ & - \\
\hline Variables & \multicolumn{2}{|c|}{$P$-Value } & SE & \multicolumn{2}{|c|}{ LSD (5\%) } & \multicolumn{2}{|c|}{ P-Value } & \multicolumn{2}{|l|}{ SE } & LSD (5\%) \\
\hline Weed Practices & \multicolumn{2}{|c|}{$\begin{array}{c}0.0000 * \\
*\end{array}$} & 0.0387 & \multicolumn{2}{|c|}{0.0776} & \multicolumn{2}{|c|}{$0.0000 * *$} & \multicolumn{2}{|c|}{0.0339} & 0.0680 \\
\hline Seed rates & \multicolumn{2}{|c|}{$\begin{array}{c}0.0000^{*} \\
*\end{array}$} & 0.0292 & \multicolumn{2}{|c|}{0.0586} & 0.00 & $0 * *$ & 0.0256 & & 0.0514 \\
\hline $\mathbf{W} \times \mathbf{S}$ & $\begin{array}{c}0.000 \\
*\end{array}$ & & 0.0774 & & .1551 & 0.0 & $2 * *$ & $0.067 \varepsilon$ & & 0.1360 \\
\hline
\end{tabular}

$\mathrm{NW}=$ No weeding, SIPE $=$ Sorghum immature plant extract, SMPE $=$ Sorghum mature plant extract, $\mathrm{HW}=$ Hand weeding, NS $=$ Non-significant at $\mathrm{P}_{0.05} ; *$ Significant at $\mathrm{P}_{0.05}$; $* *=$ Significant at $\mathrm{P}_{0.01}$.

\section{Kernel yield (t ha-1)}

This (Table 4) revealed that various weed control treatments seriously improved seed yield attributes of maize in contrast to no weeding. The compound of sorghum immature plant extract @ $15 \mathrm{~L} \mathrm{ha}^{-1}+$ herbicide (Mesotrione Atrazine) @ $1.0 \mathrm{~L} \mathrm{ha}^{-1}$ considerably produced more kernel yield (6.8 $\mathrm{t} \mathrm{ha}^{-1}$ ) followed by integration of sorghum mature plant extract @ 15 L $\mathrm{ha}^{-1}+$ herbicide (Mesotrione Atrazine) @ $1.0 \mathrm{~L} \mathrm{ha}^{-1}$ subsequent in $\left(6.4 \mathrm{t} \mathrm{ha}^{-1}\right)$. Nevertheless, lowest (4.2 $\mathrm{t} \mathrm{ha}^{-1}$ ) kernel yield was noticed at no weeding. Kernel yield was also significantly influenced by seed rates. The seed rate $35 \mathrm{~kg}$ per ha produced greater (5.8 $\mathrm{t} \mathrm{ha}^{-1}$ ) kernel yield followed by seed rates 25 and 30 $\mathrm{kg}$ per ha with 5.5 and $5.7 \mathrm{t}$ per ha and lesser ( $5.3 \mathrm{t} \mathrm{ha}^{-1}$ ) kernel yield was noted under seed rate $20 \mathrm{~kg}$ per ha. Significant interactive effects of weed control practices $\times$ seed rates were found for kernel yield $\left(t \mathrm{ha}^{-1}\right)$. Combined utilization of allelopathic plants extracts is more helpful for controlling weeds as compared to alone (Nouri et al., 2012). Weed control through allelopathic plant extract, enhanced seed yield of different crops from 15 to 25\% (Cheema et al., 2013). Our findings are in concurrence with Stougaard and Xue (2004) who concluded that plant population was more compulsory for suppression weeds development for getting better grain 
yield. Increasing seed rate from 175 plants $\mathrm{m}^{-2}$ to 280 plants $\mathrm{m}^{-2}$ improved crop production $12 \%$. Hight plant density increased financial yield and prevented the growth of other plants (Amiri et al., 2014). Gozubenli et al. (2004) reported that grain yield increased as density increased up to 9 plants $\mathrm{m}^{-2}$. Similarly, Ahmed et al. (2014) concluded that increasing seeding rates can defeat weed growth and increase grain yield.

\section{Conclusion}

The results of present research suggested that integrated application of sorghum immature plant extract @ 15 L ha ${ }^{-1}$ with $50 \%$ reduced dose of herbicide (Mesotrione Atrazine) @ $1.0 \mathrm{~L} \mathrm{ha}^{-1}$ controlled weeds and enhanced maize kernel yield remarkably. Amongst seed rates, $30 \mathrm{~kg}$ per ha was found appropriate for controlling weeds and obtaining optimal kernel yield of maize.

\section{Acknowledgments}

This research paper is arranged from
PhD dissertation of main author submitted at Sindh agriculture university Tandojam.

\section{Statement of Novelty}

The current research work examines the allelopathic effect of sorghum immature and mature plant extract in conjunction with seed rates on weed management and yield improvement in maize for the first time under agro-climatic conditions of Tandojam, Sindh. This could help in weed reduction and yield enhancement at the local surroundings.

\section{Contribution of Authors}

T.A. Keerio, design experiment, collected data and prepared document. M.N. Kandhro guided scholar as a whole from designing experiment to writing of article. A.N. Shah edited manuscript. M.I. Keerio and G. M. Jamro helped in to compile research material, data analysis and explanation of results.

\section{Conflict of interest}

There is no conflict of interest among authors. 


\section{REFERENCES CITED}

Ahmad, H., M. Shafi, W. Liaqat, M.F. Jan and W. Rehan. 2018. Effect of tillage practices and weed control methods on yield and yield components of maize. Middle East $\mathrm{J}$. Agric. Res., 7(1): 175-181.

Ahmed, S., M. Salim and B.S., Chauhan. 2014. Effect of Weed Management and Seed Rate on Crop Growth under Direct Dry Seeded Rice Systems in Bangladesh. PLoS One 9(7): e101919.

Amiri Z., A. Tavakkoli and M. Rastgoo. 2014. Responses of Corn to Plant Density and Weed Interference Period. Middle-East J. Sci. Res., 21 (10): 1746-1750

Arce, G.D., P. Pedersen and R.G. Hartzler. 2009. Soybean seeding rate effects on weed management. Weed. Technol., 23(1): 17-22.

Asad, M., Z. Mahmood, M. Mudassar, A. Arshad, M.U. Raza and W. Anum. 2019. Bio-economic assessment of non-chemical weed management strategies in minor crops: A review on Weed research issues, challenges, and opportunities in Pak. J. Weed Sci. Res., 2: 127-140.

Bastiaans, L. 2008. Focus on ecological weed management: What is hindering adoption. Weed Res., 48:481-491.

Chauhan, B.S and D.E. Johnson. 2011. Row spacing and weed control timing affect yield of aerobic rice. Field Crops Res., 121: 226-231.

Chauhan, B.S. 2020. Grand challenges in weed management. Front. Agron., $1: 3$.

Cheema, Z.A., A. Khaliq and M. Tariq. 2002. Evaluation of concentrated sorgaab alone and in combination with three pre-emergence herbicides for weed control in cotton (Gossypium hirsutum L.). Int. J. Agric. Biol., 4(4): 549-452.

Cheema, Z. A., A. Khaliq and S. Saeed. 2004. Weed control in maize (Zea mays L.) through sorghum allelopathy. J. Sustain. Agric., 23: 73-86.

Cheema, Z.A., M.N. Mushtaq, M. Farooq, A. Hussain and I.U. Din. 2009. Purple nutsedge management with allelopathic sorghum. Allelopathy J., 23: 305-312.

Cheema, Z.A., M. Farooq and A. Khaliq. 2013. Application of allelopathy in crop production: Success story from Pakistan. Allelopathy (Springer Link), 6: 113-143.

Chopra, N., G. Tewari, L.M. Tewari, B. Upret and N. Pandey. 2017. Allelopathic effect of Echinochloa colona (L.) and Cyperus iria (L.) weed extracts on the seed germination and seedling growth of rice and soybean. Adv. Agric., 4: 15.

Deka, S.J., G.C. Sarma and S.P. Deka. 2011. Allelopathic effects of weed plants on germination of herbaceous plant seeds. J. EcoBiol., 28(2): 123-130.

Farooq, N., T. Abbas, A. Tanveer and K. Jabran. 2020. Allelopathy for Weed Management. In: Co-Evolution of Secondary Metabolites Springer Link. pp. 505-519.

Gianessi, L. and A. Williams. 2011. Overlooking the obvious the opportunity for herbicides in Africa. Outlooks. Pest Manage., 211-215

Gill, M.S. 2008. Productivity of directseeded rice (Oryza sativa) under 
varying seed rates, weed control and irrigation levels. Indian J. Agri. Sci., 78(11): 766-70.

Gozubenli, H., M. Kilinc, O. Sener and O. Konuskan. 2004. Effects of single and twin row planting on yield and yield components in maize. Asian $\mathrm{J}$. Plant Sci., 3(2): 203-206

Hashim, S., K.B. Marwat, M. Saeed, M. Haroon, M. Waqar and Shahfahad. 2013. Developing a sustainable and eco-friendly weed management system using organic and inorganic mulching techniques. Pak. J. Bot., 45: 483-486.

Hozayn, M., A.A.A. Monem and E.M.A. Lateef. 2011. Crop residues, an effective tool for improving growth of wheat and suppression of some associated weeds. Allelopathy J., 27(2): 237-344.

Jan, I., S. Khan and T. Mahmood. 2021. Effects of rainwater harvesting on yield of wheat and maize crops in Pakistan. Pak. J. Agric., Agric. Eng. Vet. Sci., 37(1): 29-35.

Javaid, A., S. Shafique and S. Shafique. 2010. Herbicidal effects of extracts and residue incorporation of Datura metel against parthenium weed. Nat. Prod. Res., 24(15): 1426-1437. Javaid, A. and I.H. Khan. 2020. Potential use of Coronopus didymus in parthenium management. Pak. J. Weed Sci. Res., 26(1): 37-45.

Jhala, A. J., S.C. Shah, P. H. Rathod and H. Bhatt. 2008. Integrated effect of seed rates and weed management treatments in wheat (Triticum aestivum L.). Res. J. Agric. Biol. Sci., 4(6): 704-711

Kandhro, M.N., M.A. Ansari, A. Naqi, M. Ibrahim and H.R. Memon. 2015. Laboratory studies on the allelopathic potential of sorghum and sunflower water extract and powder against narrow-leaf summer weeds. Gomal Uni. J. Res., 31: ISSN: 1019-8180

Kandhro, M.N., A.K. Jalbani, N.A. Wahocho, G.M. Sahito, M. Solangi and Q. Jogi. 2016. Laboratory studies on germinability and seedling growth of cotton crop under the allelopathic influence of purple nutsedge. Pak. J. Weed Sci. Res., 22(3): 407-416.

Karki T, S.B. B.K., and R.C. Mishra. 2010. Critical period of weed control in maize. Nepalese J. Agric. Sci., 8: 39-47.

Khaliq A, Matloob A, Tanweer A, Khan M.B. 2012. Naturally occurring phytotoxins in allelopathic plants help reduce herbicide dose in wheat. Nat. Prod. Res., 26(12): 1156-60.

Mahmood, A. 2003. Utilization of allelopathic properties of sorghum for controlling purple nutsedge (Cyperus rotundus L.) in maize. PhD Dissertation, Univ. of Agric. Faisalabad, Pakistan.

Mahmood, A. 2009. Weed management in maize (Zea mays L.) through allelopathy. Doctor of Philosophy in Agronomy, Faculty of Agriculture, Uni. of Agri. Faisalabad, Pakistan.

Mushtaq, M.N., Z.A. Cheema and A. Khaliq. 2010. Effects of mixture of allelopathic plant aqueous extracts on (Trianthema portulacastrum L.) Weed. Allelopathy J., 25: 205-212.

Narwal, S.S. 2000. Weed management in rice wheat rotation by allelopathy. Crit. Rev. Plant Sci., 19: 246-249.

Nouri, H., Z.A. Talab and A. Tavassoli. 2012. Effect of weed allelopathic of 
526 Tariq Ahmed Keerio, Muhammad Nawaz Kandhro et al. Allelopathic Impact of ....

sorghum (Sorghum halepense) on germination and seedling growth of wheat, Alvand cultivar. Ann. Biol. Res., 3(3):1283-1293.

Rajcan, I., and C.J. Swanton. 2001. Understanding maize weed competition resource competition, light quality and the whole plant. Field Crops Res., 71: 139-150.

Sharifi, R.S., M. Sedghi and A. Gholipouri. 2009. Effect of population density on yield and yield attributes of maize hybrids. Res. J. Biol. Sci., 4(4): 375-379.

Sharifi, R.S., and A. Namvar. 2016. Plant density and intra-row spacing effects on phenology, dry matter accumulation and leaf area index of maize in second cropping. Biologia, 62(1): 46-57.

Sharma, S.N. and R.K. Singh. 2011. Seed rate and weed management on yield and nutrient uptake of wheat (Triticum aestivum). Indian $\mathrm{J}$. Agric. Sci., 81(12): 1174-9.

Stougaard, R.N. and Q. Xue. 2004. Spring wheat seed size and seeding rate effects on yield loss due to wild oat (Avena fatua) interference. Weed Sci., 52: 133-141.

Valadabadi, S.A. and H.A. Farahani. 2010. Effects of plant density and pattern on physiological growth indices in maize (Zea mays L.) under nitrogenous fertilizer application. J. Agric. Ext. Rural Dev., 2(3): 40-7.

Weston, L.A. and S.O. Duke. 2003. Weed and crop allelopathy. Critical Rev. Plant Science, 22: 367-389

Weston, L.A., I.S. Alsaadawi and S.R. Baerson. 2013. Sorghum allelopathy from ecosystem to molecule. J. Chem. Ecol., 39 (2):
142-153.

Yadav, D.P., R.D. Vaishya and G. Singh. 2001. Response of late sown wheat to method of sowing, seed rate and weed management treatments. Ann. Agric. Res., 22: 429-431.

A. 